\title{
The stellar winds of Galactic Centre and the low accretion rate of Sgr A*
}

\author{
R. F. Coker ${ }^{\star}$ \\ Department of Physics and Astronomy, University of Leeds, Leeds LS2 9JT, UK
}

Received 5 June 2001 / Accepted 19 June 2001

\begin{abstract}
An attempt is made to reconcile the large wind-loss rates of stars in the Galactic Centre (GC) with the predicted low accretion rate for Sgr $\mathrm{A}^{*}$, the putative blackhole at the heart of the Milky Way. It is found that, independent of the details of the accretion, the bound but unaccreted gas has been accumulating in the potential well of Sgr A* for $\lesssim 10^{3}$ yrs and thus is not in equilibrium. Otherwise, the gas flows of the region would be visible in both the IR and X-ray. It appears that the blackhole was more active in the recent past due to the passing of a supernova blast shock but is presently in a short-lived dormant phase. The extended low frequency radio emission from the central parsec should visibily increase over the next few decades, as the shock passes completely in front of the absorbing gas and dust near Sgr $\mathrm{A}^{*}$. The GC may become more active in $\lesssim 10^{5}$ yrs due to either another supernova or sufficient accumulation of stellar winds in the central arcsecond.
\end{abstract}

Key words. hydrodynamics - ISM: structure - stars: winds, outflows - galaxy: centre - accretion

\section{Introduction}

Sgr $\mathrm{A}^{*}$, the stationary, compact, nonthermal radio source at the Galactic Centre (GC), appears to be coincident with a $2.6 \pm 0.2 \times 10^{6} M_{\odot}$ point-like object (Eckart \& Genzel 1996, 1997; Ghez et al. 1998; Backer \& Sramek 1999; Genzel et al. 2000; Ghez et al. 2000). It is probable that $\operatorname{Sgr} \mathrm{A}^{*}$ is associated with the accretion of matter onto a supermassive blackhole (for a recent review of the evidence, see Genzel \& Eckart 1999). Models for the accretion process itself vary widely, including simplistic spherical accretion (Coker \& Melia 2000; Coker \& Markoff 2001), an advective flow (Narayan et al. 1995), a convective flow (Stone et al. 1999), a compact jet (Falcke \& Markoff 2000), and a truncated power-law electron distribution (Beckert \& Duschl 1997). The true picture is probably some combination of these various models.

Deep in the potential well of Sgr A* and pervading the central parsec of the Milky Way, there exists a cluster of a few dozen early-type stars (Sellgren et al. 1990; Genzel et al. 1996), which is dominated by the IRS 16 assemblage of probable Wolf-Rayet (WR) stars (Najarro et al. 1997). Numerous observations and models (Hall et al. 1982; Allen et al. 1990; Geballe et al. 1991; Yusef-Zadeh \& Melia 1992; Najarro et al. 1997; Hanson et al. 1998; Paumard et al. 2001) provide evidence that this cluster is producing a composite hypersonic wind of $\dot{M}_{\mathrm{w}} \gtrsim 10^{-3} \dot{M}_{\odot} \mathrm{yr}^{-1}$.

* e-mail: robc@ast.leeds.ac.uk
Some fraction of $\dot{M}_{\mathrm{w}}$ will be trapped by the supermassive blackhole located near the middle of the stellar cluster. However, all of the above models for Sgr A* require a mass accretion rate that is orders of magnitude less than $\dot{M}_{\mathrm{w}}$. If any of these models are correct, then for the lifetime of the early-type cluster's winds and in the absence of any observed large-scale outflow from Sgr A*, some part of the cluster's winds has been accumulating in the central parsec without being accreted by the blackhole. It is the purpose of this letter to investigate this gas in the light of recent X-ray observations of the GC.

\section{The mass accumulation rate}

The gas supply rate into the central few parsecs, based on stellar wind measurements, is at least $\dot{M}_{\mathrm{w}} \sim$ $10^{-3} \dot{M}_{\odot} \mathrm{yr}^{-1}$. Sgr A West is an HII region in the central parsec and contains the streamers of the "mini-spiral", while the "circumnuclear disk" (CND) is a dense, clumpy, and asymmetric ring-like feature which extends for more than 7 pc. The CND has an inner radius of $\simeq 1.5 \mathrm{pc}$ and surrounds Sgr A* and Sgr A West. Both the CND and Sgr A West may be additional sources of infalling gas (Mezger et al. 1996; Vollmer \& Duschl 2001). The Bar, part of the "mini-spiral" and located $\sim 0.1$ pc south of Sgr A*, may be intercepting some fraction of the cluster's winds. In sum, $\dot{M}_{0} \sim 10^{-3} \dot{M}_{\odot} \mathrm{yr}^{-1}$ is a conservative lower limit to the total rate which gas is being supplied 
to the central parsecs. Most of this gas, in the absence of shocks and subsequent radiative cooling, is unbound and will carve out a central cavity within Sgr A West. A fraction of $\dot{M}_{0}, \dot{M}_{\mathrm{A}}$, is trapped but not necessarily accreted by $\operatorname{Sgr} \mathrm{A}^{*}$.

Assuming static spherical wind sources and calculating the fraction of each star's wind that is captured by Sgr A*, Quataert et al. (1999) estimated that $\dot{M}_{\mathrm{A}} \gtrsim$ $10^{-5} M_{\odot} \mathrm{yr}^{-1}$. A capture radius can be defined by

$R_{\mathrm{A}} \equiv \frac{2 G M}{v_{\mathrm{w}}^{2}} \simeq 0.04 \mathrm{pc} \simeq 10^{5} R_{\mathrm{s}} \simeq 1^{\prime \prime}$,

and the Schwarzschild radius by

$R_{\mathrm{s}} \equiv 2 G M / c^{2}$,

where $c$ is the speed of light and $v_{\mathrm{w}}\left(\sim 700 \mathrm{~km} \mathrm{~s}^{-1}\right)$ is the velocity of the supersonic wind flowing past a centralized object of mass $M$ (taken to be $\left.2.6 \times 10^{6} M_{\odot}\right)$. Stellar motions, wind-wind collisions, radiative cooling, and other unidentified wind sources may substantially alter this estimate. In addition, the mass-loss rates of the cluster members may have been overestimated by a factor of a few (Morris et al. 2000). The rotation of the cluster stars around Sgr A* (Genzel et al. 2000) may result in angular momentum support of their winds (but see below) and the strong magnetic fields in the central parsec may also play a role in supporting the gas against accretion. Nonetheless, it appears that $\dot{M}_{\mathrm{A}} \sim 10^{-5} M_{\odot} \mathrm{yr}^{-1}$ is a good estimate of the minimum amount of gas that is being trapped in the central arcsecond by the potential well of Sgr A*.

According to all of the accretion models for $\operatorname{Sgr} \mathrm{A}^{*}$, the actual accretion rate, $\dot{M}$, onto the blackhole is much less than $\dot{M}_{\mathrm{A}}$. In fact, the accretion rate through a radius of $R_{\min } \sim 1$ mas is thought to be orders of magnitude smaller than the above estimate for $\dot{M}_{\mathrm{A}}$. There is no evidence of any outflow from $\mathrm{Sgr} \mathrm{A}^{*}$ on scales larger than $R_{\text {min }}$, so even the presence of a mini-jet or similar small-scale outflow would not alter the fact that $\gtrsim 10^{-5} M_{\odot} \mathrm{yr}^{-1}$ is being trapped - but, seemingly, not accreted - by the gravitational potential of the blackhole. If the wind sources are WR stars as suggested by Tamblyn et al. (1996) (but see below), with a WR wind lifetime of $t_{\mathrm{w}} \sim 10^{5}$ yrs (Maeder \& Meynet 1987), the total mass of gas that has accumulated between $R_{\mathrm{A}}$ and $R_{\text {min }}$ is $\sim \dot{M}_{0} t_{\mathrm{w}} / 30=\dot{M}_{\mathrm{A}} t_{\mathrm{w}} \equiv M_{\mathrm{g}} \simeq 3 M_{\odot}$.

Recent Chandra X-ray observations (Baganoff et al. 2001) show that the average ionized gas density $\sim 1 R_{\mathrm{A}}$ from $\mathrm{Sgr} \mathrm{A}^{*}$ is only $\sim 10^{2} \mathrm{~cm}^{-3}$. If $M_{\mathrm{g}}$ has accumulated within $R_{\mathrm{A}}$, then the average number density of the captured gas is $\bar{n} \sim 10^{4} \mathrm{~cm}^{-3}$. The most likely explanation for this discrepancy is that $t_{\mathrm{w}} \ll 10^{5} \mathrm{yrs}$ and the GC is presently in a state of quiesence.

\section{The cyclic feeding of Sgr A*}

A few million years ago, the GC underwent a burst of star formation (Tamblyn et al. 1996) which resulted in a number of clusters of massive stars (IRS 16, the Arches Cluster, and the Quintuplet Cluster). Remnants of this mini-starburst can still be seen in GC star-forming regions such as the "50 $\mathrm{km} \mathrm{s}^{-1}$ cloud" (Serabyn et al. 1992; Mezger et al. 1989). The central early-type cluster may have formed more than a parsec away from $\mathrm{Sgr} \mathrm{A}^{*}$ and then, over a million years, spiraled inward via dynamical friction (Gerhard 2001). The disrupted remnant of the parent molecular cloud may be seen as the "ionized gas halo" which fills the central 10 parsecs (Anantharamaiah et al. 1999).

Then, $\sim 10^{4}$ yrs ago, a $13-20 M_{\odot}$ star, located $\sim 5$ pc east of $\operatorname{Sgr} \mathrm{A}^{*}$, exploded in a mixed-morphology type II supernova (SN), sweeping up the "ionized gas halo" and producing what we now see as Sgr A East (Maeda et al. 2000). The eastern edge of the explosion has been confined by the "50 $\mathrm{km} \mathrm{s}^{-1}$ cloud" while the western edge has interacted with Sgr A* and the central cluster. Indeed, a few thousand years ago, after overwhelming the winds from the mass-losing stars of the early-type cluster, the dense frontal shock of the explosion swept over Sgr A*, triggering a period of high accretion rate and X-ray luminosity for the blackhole (Baganoff et al. 2001).

This period of high accretion lasted until the ram pressure of the front shock near Sgr A* dropped beneath that of the central early-type cluster's winds $\left(\sim 10^{(-7)-(-6)}\right.$ dynes $\left.\mathrm{cm}^{-2}\right)$. Based on SN models by Pittard et al. (2001), this "binging" by Sgr A* lasted $10^{2-3}$ yrs. For the last thousand years or so, after the passage of the dense shell, the IRS 16 and other early-type stars have been "purging" the central parsecs of the hotter, less dense post-shock SN cavity material. Sgr A West is $\sim 1 \mathrm{pc}$ in radius, consistent with the volume these stars could have cleared in $\sim 10^{3}$ yrs while the arms of the "minispiral" are tendrils of infalling gas from this inherently unstable process. Thus, $t_{\mathrm{w}} \sim 10^{3}$ yrs and $\bar{n} \sim 10^{2} \mathrm{~cm}^{-3}$, consistent with the X-ray observations. The lower density also reduces the expected IR thermal bremsstrahlung emission from the central arcsecond to below that which is observed (Menten et al. 1997). Note that radiation pressure from the early-type cluster is not likely to influence the accumulation of gas in the central arcsecond. For radiation pressure to be significant, the luminosity of the cluster, estimated to be $\lesssim 10^{8} L_{\odot}$, would need to be larger than the Eddington luminosity for Sgr A*. This is almost certainly not the case (Latvakoski et al. 1999).

\section{Discussion}

Since the trapped gas is not actually accreting onto Sgr $A^{*}$, it implies that dissipation of angular momentum in the central arcsecond is, at present at least, inefficient. Over the next $10^{5} \mathrm{yrs}$, the density of the ISM in the central arcsecond will increase as the winds from the earlytype cluster fill the region. Eventually, sufficient dissipation may result in the formation of a more standard accretion disk, turning our Galaxy into a low level Seyfert. However, if the stars in the central few parsecs formed at 
the same time, it is likely that another SN will explode in less than $10^{5} \mathrm{yrs}$, truncating the accumulation process and triggering another "binge and purge" cycle.

If, as recently proposed (Paumard et al. 2001), the early-type cluster stars are primarily Luminous Blue Variables (LBVs) rather than WR stars, the shorter duration of the LBV phase would imply that the cluster has been producing massive winds for $\lesssim 10^{4}$ yrs. However, the winds from LBVs are slower $\left(\sim 200 \mathrm{~km} \mathrm{~s}^{-1}\right)$ and more massive $\left(\sim 10^{-3} \dot{M}_{\odot} \mathrm{yr}^{-1}\right)$ than WR winds, with a resulting value of $\bar{n}$ that is still $\sim 50$ times larger than the previous estimate which assumed the wind sources to be WR stars. Nonetheless, if the IRS 16 stars have entered the LBV phase only within the last few centuries, the mass of trapped gas would be sufficiently reduced to meet the $\mathrm{X}$ ray observational limits. If so, Sgr A* might become significantly brighter in the next few centuries as the LBV winds continue to fill the accretion region. However, IRS 7, a red supergiant $\simeq 0.3 \mathrm{pc}$ from Sgr $\mathrm{A}^{*}$, has a tail that points away from IRS 16 and Sgr A*. Models (Yusef-Zadeh \& Melia 1992; Dyson \& Hartquist 1994) show that a GC wind of $\sim 500 \mathrm{~km} \mathrm{~s}^{-1}$ is needed to produce this tail. Such a wind would require $\sim 500$ yrs to reach IRS 7 from IRS 16, implying that the wind sources are not exclusively LBVs.

Another possibility is that the gas flows in the central few arcseconds are not in equilibrium due to an explosive event $\sim 10^{3-4}$ yrs ago. As mentioned above, Sgr A East is a probable mixed-morphology type II supernova remnant (SNR) that appears to envelope Sgr A West, the compact HII region which contains both $\mathrm{Sgr} \mathrm{A}^{*}$ and the early-type cluster (Goss et al. 1989). When the progenitor of Sgr A East, located $\sim 5 \mathrm{pc}$ from Sgr $\mathrm{A}^{*}$, exploded $\sim 10^{4}$ yrs ago, the strong frontal shock could have cleared the central parsec of its accumulation of wind as it swept by Sgr A* $\sim 10^{3}$ yrs ago (Maeda et al. 2001). With a temperature of $2 \mathrm{keV}$ and a density of $\sim 10 \mathrm{~cm}^{-3}$ (Maeda et al. 2001), the pressure of the rarified gas in the cavity of the SNR is $\lesssim 10^{-7}$ dyne $\mathrm{cm}^{-2}$ while the ram pressure due to the wind from an individual cluster member at a distance of $R_{\mathrm{A}}$ is on average $\gtrsim 10^{-6}$ dyne $\mathrm{cm}^{-2}$. Thus, after the passage of the dense frontal shock, the cluster wind would have overwhelmed the gas in the SNR cavity and refilled the central $R_{\mathrm{A}}$ over the last $\sim 10^{3}$ yrs. Sgr B2, an X-ray reflection nebula near the GC, seems to have been illuminated by Sgr A* $\sim 500$ yrs ago, suggesting that the frontal shock passed by Sgr $A^{*}$ at that time, dramatically increasing the emission of the blackhole (Sunyaev et al. 1993; Koyama et al. 1996; Murakami et al. 2000). The frontal shock was likely to be slow and dense enough to result in Sgr A* trapping enough gas to be accreting at near its Eddington rate for $\sim 10^{3}$ yrs (Maeda et al. 2001; Baganoff et al. 2001).

Since IRS 7 is presently interacting with the wind from IRS 16 , the central $R_{\mathrm{A}}$ is likely to be filled with IRS 16 winds. Thus, the rarified cavity wind from Sgr A East is unlikely to be the present source of accretion for $\operatorname{Sgr} \mathrm{A}^{*}$ as suggested by Baganoff et al. (2001). However, if IRS 7 is interacting with a wind from Sgr A* rather than IRS 16, it would be evidence of recent activity near the black- hole, suggesting that the IRS 16 winds have been reasserting themselves only in the last few centuries, if at all. A continual outflow from Sgr A* rather than IRS 16 would clear the central arcsecond of gas but would require a fast and/or dense outflow on scales greater than $R_{\min }$; there is no observational evidence of such a wind.

In any case, IRS 16C, a WR star only a few arcseconds from Sgr $\mathrm{A}^{*}$, is alone producing a sufficient wind to accumulate almost $10^{-5} M_{\odot} \mathrm{yr}^{-1}$ within $R_{\mathrm{A}}$. Assuming the blackhole is accreting only a fraction of this, the upper limit on the time the wind from this star has been accumulating in the central arcsecond is $10^{4} \mathrm{yrs}$. Since there are dozens of young massive stars with heavy winds in the central parsec, it is likely that $t_{\mathrm{w}} \lesssim 10^{3}$ yrs.

It is thought that Sgr A West and Sgr A* lie only slightly in front of Sgr A East since the $90 \mathrm{~cm}$ emission from the expanding shell of Sgr A East is seen in absorption at the location of Sgr A West (Yusef-Zadeh et al. 1999). However, a north-south tongue of emission which cuts through Sgr A West and approaches Sgr A*, can be seen. If the proposed scenario is correct, this tongue corresponds to the leading edge of the SNR and thus should expand over the next few decades as the shell moves more completely in front of Sgr A West.

It has been assumed that the early-type cluster is not displaced from Sgr A* by more than a few arcseconds along the line of sight. Proper motion and radial velocity observations (Genzel et al. 2000) support this and in fact suggest the orbits of the cluster stars are nearly Keplerian. If cluster members are on Keplerian orbits around Sgr A*, their orbital velocities might be an appreciable fraction of their wind velocities. This may add sufficient angular momentum to the winds so that a larger fraction of the gas never reaches the capture radius. However, the density of ionized gas falls off outside $\sim 1^{\prime \prime}$ (Baganoff et al. 2001), suggesting that only a fraction of $\dot{M}_{\mathrm{A}}$ is being trapped outside the capture radius due to support from angular momentum or magnetic fields.

\section{Conclusions}

A fraction of the winds from the few dozen early-type stars in the central parsec of the GC is trapped by the potential well of Sgr A* but is not currently being accreted by the blackhole. Unlike an isolated cluster, this gas has insufficient ram pressure or thermal pressure to escape so that gas has been accumulating in the central $R_{\mathrm{A}} \sim 1^{\prime \prime}$ of the Galaxy. Independent of the details of the flow, the estimated density of this gas is inconsistent with IR and X-ray observations unless the accumulation has been proceeding for $\lesssim 10^{3}$ yrs. This implies the gas flows in the central $\sim 1^{\prime \prime}$ are not in equilibrium and the present low mass accretion rate onto Sgr $\mathrm{A}^{*}$ is a short-lived phase.

Due to the tendency to form more high mass stars when deep in a potential well, as well as the early-type stars from the present mini-starburst, the SN rate in the GC is thought to be $\gtrsim 10^{-5} \mathrm{yr}^{-1}$ (Tamblyn \& Rieke 1993). In addition, starbursts may recur in the GC every 
$\sim 10^{7-8}$ yrs (Ozernoy 1994; Morris et al. 1999). If so, within each cycle of star formation, the sub-cycle of "binge and purge" may repeat on a time-scale of $\lesssim 10^{5}$ yrs. This episodic behaviour should appear in any galaxy with star formation near a central supermassive blackhole. Similar feedback mechanisms have been proposed for elliptical galaxies (Ciotti \& Ostriker 2001).

In the case of the GC, one would then expect that 1) the $90 \mathrm{~cm}$ emission near $\mathrm{Sgr} \mathrm{A}^{*}$ to visibily brighten over the next few decades as the shell of the SNR moves completely in front of Sgr A West, 2) the emission from Sgr A* itself should increase over the next $\gtrsim 10^{3}$ yrs at all frequencies as the cluster's winds again accumulate, and 3 ) a SN should detonate in the next $\lesssim 10^{5}$ yrs, leading to a brief $\left(\sim 10^{3} \mathrm{yrs}\right)$ period of high accretion and the start of the next "binge and purge" cycle.

Acknowledgements. This work was supported by PPARC and has made use of NASA's Astrophysics Data System Abstract Service. I would like to thank J. M. Pittard for many useful discussions and S. Markoff and B. A. Cohen for comments on the manuscript.

\section{References}

Allen, D., Hyland, A., \& Hillier, D. 1990, MNRAS, 244, 706

Anantharamaiah, K. R., Pedlar, A., \& Goss, W. M. 1999, in The Central Parsecs of the Galaxy, ed. H. Falcke, A. Cotera, W. Huschl, F. Melia, \& M. Rieke, ASP Conf. Ser., 186, 422

Backer, D. C., \& Sramek, R. A. 1999, ApJ, 524, 805

Baganoff, F. K., Maeda, Y., Morris, M., et al. 2001, ApJ, in press

Beckert, T., \& Duschl, W. 1997, A\&A, 328, 95

Ciotti, L., \& Ostriker, J. P. 2001, ApJ, 551, 131

Coker, R. F., \& Markoff, S. 2001, in Galaxies and their Constituents at the Highest Angular Resolution, IAU Symp., 205, 43

Coker, R. F., \& Melia, F. 2000, ApJ, 534, 723

Dyson, J. E., \& Hartquist, T. W. 1994, MNRAS, 269, 447

Eckart, A., \& Genzel, R. 1996, Nature, 383, 415

Eckart, A., \& Genzel, R. 1997, MNRAS, 284, 576

Falcke, H., \& Markoff, S. 2000, A\&A, 362, 113

Geballe, T., Krisciunas, K., Bailey, J., \& Wade, R. 1991, ApJ, 370, L73

Genzel, R., \& Eckart, A. 1999, in The Central Parsecs of the Galaxy, ed. H. Falcke, A. Cotera, W. Huschl, F. Melia, \& M. Rieke, ASP Conf. Ser., 186, 3

Genzel, R., Pichon, C., Eckart, A., Gerhard, O. E., \& Ott, T. 2000, MNRAS, 317, 348

Genzel, R., Thatte, N., Krabbe, A., Kroker, H., \& TacconiGarman, L. 1996, ApJ, 472, 153
Gerhard, O. 2001, ApJ, 546, L39

Ghez, A. M., Klein, B. L., Morris, M., \& Becklin, E. E. 1998, ApJ, 509, 678

Ghez, A. M., Morris, M., Becklin, E. E., Tanner, A., \& Kremenek, T. 2000, Nature, 407, 349

Goss, W. M., Anantharamaiah, K. R., van Gorkom, J. H., et al. 1989, in IAU Symp. 136: The Center of the Galaxy, 136, 345

Hall, D., Kleinmann, S., \& Scoville, N. 1982, ApJ, 260, L53

Hanson, M., Rieke, G., \& Tamblyn, P. 1998, BAAS, 193, 104.02

Koyama, K., Maeda, Y., Sonobe, T., et al. 1996, PASJ, 48, 249

Latvakoski, H. M., Stacey, G. J., Gull, G. E., \& Hayward, T. L. 1999, ApJ, 511, 761

Maeda, Y., Baganoff, F., Feigelson, E., et al. 2000, BAAS, 197, 80.03

Maeda, Y., Baganoff, F. K., Feigelson, E. D., et al. 2001, ApJ, in press

Maeder, A., \& Meynet, G. 1987, A\&A, 182, 243

Menten, K. M., Reid, M. J., Eckart, A., \& Genzel, R. 1997, ApJ, 475, L111

Mezger, P., Duschl, W., \& Zylka, R. 1996, A\&AR, 7, 289

Mezger, P. G., Zylka, R., Salter, C. J., et al. 1989, A\&A, 209, 337

Morris, M., Ghez, A. M., \& Becklin, E. E. 1999, Adv. Space Res., 23, 959

Morris, P. W., van der Hucht, K. A., Crowther, P. A., et al. 2000, A\&A, 353, 624

Murakami, H., Koyama, K., Sakano, M., Tsujimoto, M., \& Maeda, Y. 2000, ApJ, 534, 283

Najarro, F., Krabbe, A., Genzel, R., et al. 1997, A\&A, 325, 700

Narayan, R., Yi, I., \& Mahadevan, R. 1995, Nature, 374, 623

Ozernoy, L. M. 1994, BAAS, 185, 66.05

Paumard, T., Maillard, J. P., Morris, M., \& Rigaut, F. 2001, A\&A, 366, 466

Pittard, J. M., Dyson, J. E., Falle, S. A. E. G., \& Hartquist, T. W. 2001, A\&A, in press

Quataert, E., Narayan, R., \& Reid, M. J. 1999, ApJ, 517, L101

Sellgren, K., McGinn, M. T., Becklin, E. E., \& Hall, D. N. 1990, ApJ, 359, 112

Serabyn, E., Lacy, J. H., \& Achtermann, J. M. 1992, ApJ, 395, 166

Stone, J. M., Pringle, J. E., \& Begelman, M. C. 1999, MNRAS, 310,1002

Sunyaev, R. A., Markevitch, M., \& Pavlinsky, M. 1993, ApJ, 407, 606

Tamblyn, P., Rieke, G., Hanson, M., et al. 1996, ApJ, 456, 206

Tamblyn, P., \& Rieke, G. H. 1993, ApJ, 414, 573

Vollmer, B., \& Duschl, W. J. 2001, A\&A, 367, 72

Yusef-Zadeh, F., \& Melia, F. 1992, ApJ, 385, L41

Yusef-Zadeh, F., Stolovy, S. R., Burton, M., et al. 1999, in The Central Parsecs of the Galaxy, ed. H. Falcke, A. Cotera, W. Huschl, F. Melia, \& M. Rieke, ASP Conf. Ser., 186, 197 